\title{
L'étayage entre pairs comme aide à la communication en classe de français, langue étrangère
}

\author{
Muller, Catherine \\ Université Paris 3 - Sorbonne Nouvelle \\ DILTEC \\ catherine.muller9@gmail.com
}

\section{Introduction}

Parler d' " étayage entre pairs » implique d'avoir opéré un certain nombre de glissements de sens à partir du concept original d'étayage développé par Bruner (1983). Ces modifications portent d'abord sur le type d'apprentissage. L'assistance fournie au novice par une personne compétente, analysée par Vygotski puis reprise par Bruner, concerne le développement de l'enfant. Ce concept, introduit dans l'appropriation de la langue première, a ensuite été étendu à l'enseignement-apprentissage des langues (voir notamment Bange, 1996). On observe également une réappropriation du concept d'étayage concernant la personne compétente qui soutient l'apprentissage du novice. Du parent dans le cadre du développement de l'enfant, l'expert est ensuite devenu l'enseignant puis enfin un pair. Il sera nécessaire de prendre en compte la genèse de ce concept avant d'étudier ses manifestations dans un corpus recueilli en cours de français enseigné comme langue étrangère en contexte homoglotte. Alors que l'apprentissage collaboratif constitue bien souvent une caractéristique du travail en petits groupes (Hugon \& Le Cnuff, (éd.) 2011), il apparaît ici dans une activité de commentaire de photographies impliquant l'ensemble des apprenants. Ceux-ci sont amenés à solliciter de l'aide et à en apporter à leurs pairs lorsque des obstacles à la communication apparaissent. En raison de lacunes lexicales, le message ne « passe » pas. Un basculement se produit alors d'une focalisation sur le contenu à une focalisation sur la langue (Bange, 1992). Après avoir replacé la notion d'apprentissage collaboratif dans le contexte socioconstructiviste de l'étayage, nous présenterons le cadre méthodologique. Les manifestations de l'étayage entre pairs seront analysées dans un troisième temps.

\section{Genèse du concept d'étayage entre pairs}

C'est dans le cadre de l'approche socioconstructiviste que s'inscrit la notion d'étayage. Nous présenterons les caractéristiques de ce concept, ainsi que son introduction dans l'enseignement des langues. Le concept d'étayage entre pairs sera ensuite explicité.

\subsection{L'approche socioconstructiviste : la relation entre interaction et acquisition}

L'approche socioconstructiviste postule l'existence d'un lien étroit entre acquisition et interaction. L'apprentissage est envisagé comme un processus interactif qui se construit dans la relation avec autrui. Pour Vygotski, l'interaction ne se limite pas à un "simple déclencheur des processus cognitifs » (Mondada \& Pekarek Doehler, 2000 : 149), mais constitue « un facteur qui structure la forme et le contenu du développement cognitif aussi bien que langagier » (ibid). Il est nécessaire pour Bruner (1983 : 282) de prendre en considération les contextes interactifs dans le cadre de l'acquisition du langage. Celleci apparaît « dans un contexte de « dialogue d'action » dans lequel une action est entreprise conjointement par l'enfant et l'adulte» (Bruner, 1983 : 202). Les processus communicatifs sont ainsi appréhendés «comme lieu et support de l'acquisition » (Cambra Giné, 2003 : 129). On retrouve cette même conception dans la perspective interactionniste sur l'acquisition des langues secondes. Pour Py, l'acquisition constitue un processus dans lequel entre en compte « la coopération entre un apprenant et un interlocuteur plus compétent » (1996: 106). 


\subsection{La notion d'étayage}

Si l'étayage désigne toujours l'aide apportée par l'expert au novice, il doit cependant réunir certaines conditions pour être efficace.

\subsubsection{L'aide apportée par l'expert au novice}

Dans Pensée et langage ([1933] 1997), Vygotski met en évidence l'importance fondamentale de la collaboration d'un expert ou d'un pair plus compétent dans le développement de l'enfant :

l'élément central pour toute la psychologie de l'apprentissage est la possibilité de s'élever dans la collaboration avec quelqu'un à un niveau intellectuel supérieur, la possibilité de passer, à l'aide de l'imitation, de ce que l'enfant sait faire à ce qu'il ne sait pas faire ([1933] $1997: 355)$.

Grâce ce soutien, l'enfant aboutit à une meilleure performance que lorsqu'il réalise ce travail en autonomie (Vygotski, [1933] 1997 : 353). Ce phénomène a été caractérisé sous la notion d'étayage par Bruner (1983) dans le cadre des interactions d'assistance de l'adulte à l'enfant. L'étayage de l'adulte permet à l'enfant d'accomplir des tâches qu'il n'aurait pu réaliser tout seul (Bruner, 1983: 290). Ce soutien consiste à limiter la difficulté de la tâche (Bruner, $1983: 288)$ : «l'interactant plus compétent donne à l'apprenant le moyen de résoudre le problème sur lequel il bute » (Bange, $1991: 63$ ). Ce faisant, le novice acquiert des compétences (Mondada \& Pekarek Doehler, $2000: 150$ ).

\subsubsection{Les conditions de réussite de l'étayage}

Cependant, pour que l'assistance fournie par le locuteur compétent soit efficace, plusieurs conditions doivent être réunies. L'étayage ne sera fructueux que s'il est adapté au niveau de compétence de l'enfant, c'est-à-dire à la «zone prochaine de développement ${ }^{1}$ (Vygotski, [1933] 1997). Vygotski utilise ce concept pour désigner le décalage entre le niveau de développement de l'enfant lorsqu'il résout des tâches de façon autonome et son niveau lorsqu'il bénéficie de la collaboration d'un membre plus compétent ([1933] 1997: 351). Le psychologue précise: «Enseigner à l'enfant ce qu'il n'est pas capable d'apprendre est aussi stérile que lui enseigner ce qu'il sait déjà faire tout seul » (Vygotski, [1933] 1997 : 360). Même en collaboration, l'enfant ne pourra pas résoudre des problèmes situés au-delà de cette zone prochaine de développement (Vygotski, [1933] 1997 : 353). Cette «marge limitée, soumise à des règles strictes, [...] définit l'écart entre le travail autonome et le travail en collaboration » (ibid.). La tâche doit ainsi être située dans l'intervalle entre le seuil inférieur et le seuil supérieur d'apprentissage. Ce faisant, elle permet de devancer le développement, tout en étant accessible à l'enfant (Vygotski, [1933] 1997 : $355,358)$. Bruner désigne par « format » $(1983: 289)$ cette adaptation de l'aide aux capacités de l'enfant, condition de réussite de la tutelle.

En second lieu, l'aide apportée par la personne compétente doit viser l'autonomie du novice (Bange, 1996 : 191). Ce dernier pourra ainsi progressivement se passer de tutelle, en utilisant «tout seul les résultats de son ancienne collaboration» (Vygtoski, [1933] 1997: 365). Vygotski précise : «ce que l'enfant sait faire aujourd'hui en collaboration avec quelqu'un, il sera demain en état de le réaliser tout seul » ([1933] 1997 : 374). Pour que l'étayage contribue à l'autonomie de l'enfant ou de l'apprenant, il est nécessaire que «l'interactant plus compétent laisse l'apprenant agir en le guidant» (ibid.) et l'implique «dans le déroulement de la tâche» (Bange, 1996: 193). C'est à cette condition que l'apprenant comprendra «quelle action doit avoir lieu avec quel objet à quel moment et dans quel but » (ibid.). Cette « définition commune de la situation » (Bange, 1996: 192) est nécessaire pour que l'étayage porte ses fruits : « la compréhension de la solution doit précéder sa production » (Bruner, $1983: 263)$; «l'apprenti doit être capable de reconnaître une solution d'une classe déterminée de problèmes avant d'être capable lui-même de produire les démarches qui y conduisent sans aide » (ibid.). 


\subsection{L'étayage dans l'apprentissage des langues}

La notion d'étayage a été introduite dans le domaine de l'apprentissage des langues (van Lier, [1988] 1990 ; van Lier, 1996). La coopération peut se réaliser en contexte non guidé entre alloglotte et natif. On retrouve de telles interactions tutoriales dans la communication exolingue, lorsqu'un locuteur compétent fournit une aide linguistique à un locuteur alloglotte. Ici encore, pour que l'étayage soit efficace, il doit viser l'autonomie du locuteur non natif et constituer ainsi « une aide à l'autorégulation » (Bange, 1996 : 192). Dans le cadre de tandems linguistiques, chacun des partenaires est « tour à tour expert et novice, professeur et élève » (Springer, 2002: 7). Cette réciprocité repose sur les compétences des locuteurs natifs.

Le processus est similaire en classe de langue, où l'enseignant est amené à laisser l'apprenant agir tout en le guidant. Dans un contexte d'apprentissage guidé, ce concept

représente le type d'aide que peut fournir le professeur afin que l'apprenant se débrouille malgré ses manques, c'est-à-dire qu'il réalise quelque chose qu'il ne pourrait pas faire sans cette aide, ou, dit encore d'une autre façon, qu'il apprenne à faire seul ce qu'il a d'abord fait interactivement (Cambra Giné, 2003 : 126).

L'étayage consiste ainsi à «ouvrir un chemin et non [à] imposer un résultat, [à] ouvrir un chemin sur lequel l'apprenant s'avancera tant bien que mal» (Bange, 1996: 193). Le professeur peut inciter l'étudiant à la prise de risque et à l'élaboration d'hypothèses (Cambra Giné, 2003 : 127). En encourageant la résolution des difficultés de communication, il crée des conditions favorables à l'acquisition (Cambra Giné, 2003 : 138).

\subsection{L'apprentissage entre pairs}

Un étayage entre locuteurs non natifs (Ellis, [1994] 2008 : 538) est également possible. On s'intéressera ici à l'étayage entre alloglottes «d'un niveau plus ou moins semblable» (Cambra Giné, 2003 : 128). Cambra Giné souligne l'intérêt d'un «mécanisme d'échange [...] entre partenaires » (2003: 70) et d'une «dynamique d'entraide et d'étayage [...] des pairs» (2003: 138) dans la création de conditions favorables à l'acquisition en classe de langue. Cet étayage relève de l'apprentissage collaboratif, qui « ancre l'activité/l'apprentissage dans un contexte social de solidarité, d'entente, dans un véritable agir communicationnel » (Springer, 2009 : 31). Donato (1994) met en évidence l'assistance mutuelle apportée par l'étayage collectif.

La plupart des travaux sur l'apprentissage entre pairs portent sur le travail en groupes. Nussbaum souligne une hausse des «capacités de production et d'interaction » (1999: 35) grâce aux processus coopératifs favorisant le partage de connaissances entre pairs. Chaque apprenant bénéficie d'occasions de prises de parole plus importantes (Nussbaum, 1999: 36). La symétrie entre les apprenants favorise la gestion de l'interaction (Cots et al., $1997:$ : 82) et l'émergence « d'idées gérées de manière coopérative » (Nussbaum, 1999 : 36). L'achèvement interactif des énoncés (Cots et al., 1997 : 90) est également typique du travail en groupes. La possibilité d'un apprentissage coopératif réside dans le fait que «les compétences des apprenants ne sont jamais identiques et, partant, les individus peuvent endosser de manière alternée des rôles d'expert » (Nussbaum, $1999: 50$ ).

Dans les interactions en classe de langue, tout locuteur est susceptible d'être à la source du savoir. Les apprenants communiquent entre eux et un apprentissage mutuel est possible. Cicurel souligne l'importance d'« apprendre avec les autres (ou par les autres) » (2002:154) à travers les « tentatives d'un pair », les « essais et [1] es corrections données à un compagnon » (ibid.). Notre propos concernera ici plus particulièrement les aides directes apportées par les apprenants en direction de leurs pairs. Dans de telles séquences, «l'expertise «change de mains»» (Moore \& Simon, 2002: 122) : elle s'exerce entre les apprenants. C'est alors un étudiant qui endosse « le rôle de tuteur, en aidant ses condisciples à accomplir une tâche scolaire » (Lantolf \& Genung, $2000: 105$ ). 


\section{3 Élaboration du corpus et méthodes d'analyse}

Après avoir précisé les modalités d'élaboration du corpus, nous indiquerons les méthodes d'analyse adoptées.

\subsection{Constitution du corpus}

L'élaboration du corpus repose sur notre participation au dispositif mis en place (Muller, 2011a). Lors du recueil des données, la participation prend le pas sur l'observation. Il s'agit en ce sens d'une «participation observante » (Arborio \& Fournier, [1999] 2010 : 32 ; Soulé, 2007 ; Blanchet, 2011 : 73). Cette intervention dans le dispositif implique un statut double d'observateur et d'observé ${ }^{2}$. Notre perspective diffère de l'observation de classe où le chercheur n'intervient pas dans le cours, approche la plus courante dans l'analyse des interactions en classe de langue (Cicurel, 2011 : 324). Ici, le professeur anime des activités et ce sont les interactions enregistrées dans ce cadre puis transcrites qui constituent le corpus à étudier.

Ce choix inhabituel se justifie par notre volonté de mettre en place une technique spécifique dans laquelle des photographies d'auteur sont utilisées comme déclencheur d'interactions. Des groupes d'apprenants sont invités à commenter diverses photographies. L'enseignante accorde aux étudiants la possibilité de porter eux-mêmes la dynamique des échanges, ce qui se traduit par des prises de parole autosélectionnées sans régulation systématique du professeur. La parole peut alors circuler entre les étudiants de façon relativement libre. Cependant, l'enseignante doit parfois relancer les échanges ou désigner les participants afin que tous puissent s'exprimer. Au cours de cette activité, le professeur joue le rôle d'animateur (Muller, 2011b), sans chercher à focaliser l'attention sur des objectifs linguistiques prédéterminés ou à transmettre des connaissances sur l'art photographique et les dimensions culturelles.

L'intervention dans le dispositif a permis de gérer les différents paramètres et tout particulièrement celui du rôle de l'enseignant. Il aurait été difficile de trouver un professeur qui proposerait exactement cette activité, tout autant que de demander à un enseignant de mettre en place cette technique particulière. Grâce à ce positionnement double, nous avons pu disposer d'une histoire interactionnelle commune avec les apprenants et bénéficier ainsi d'une meilleure connaissance des étudiants. Comme le précise Traverso, « [a]nalyser des conversations auxquelles on a participé présente le gros avantage de réduire la part de reconstitution des données manquantes » $(1996: 3)$.

L'activité a été réalisée dans des cours de français enseigné comme langue étrangère à Paris dans un contexte associatif et universitaire avec 98 apprenants adultes de 43 nationalités répartis en 8 groupes de niveau A1 à B2. Nous avons sélectionné une douzaine de photographies d'auteur en noir et blanc pour leur qualité artistique, leur potentiel expressif et leur ambiguïté. Le corpus recueilli comporte 51 séquences de commentaires de photographies, dont la durée varie de 5 à 25 minutes, et 3 séquences de retour réflexif des apprenants.

Nous nous concentrerons ici sur un groupe de niveau A1 suivant des cours dans un contexte universitaire. Il comporte 11 apprenants ( 7 femmes et 4 hommes) âgés de 20 à 40 ans, l'âge moyen étant de 27 ans. Parmi eux, 6 sont originaires d'Amérique latine, 2 d'Europe de l'Est, 2 d'Asie de l'Est et un de Turquie. Au début des cours, 10 d'entre eux sont en France depuis moins de deux mois. Parmi les 11 étudiants, 10 ont fait des études supérieures. Nous avons recueilli 7 séquences auprès de ces apprenants au cours desquels ils commentent chaque fois une autre photographie.

\subsection{Méthodes d'analyse}

Notre approche est empirico-inductive (Blanchet, 2000 : 27). Elle pose une primauté des données, de sorte que les questions de recherche et les constructions théoriques émergent des phénomènes empiriques (Bigot, 2005 : 43 ; Allwright \& Bailey, 1991 : 36). Une telle démarche, qualitative et compréhensive, consiste à «s'interroger sur le fonctionnement et sur la signification de phénomènes humains qui éveillent la curiosité du chercheur, à rechercher des réponses dans les données » (Blanchet, $2000: 30$ ). 
C'est le phénomène d'étayage entre pairs qui a ici retenu notre attention : comment ce processus est-il utilisé par les locuteurs comme aide à la communication? Bien que l'activité ne soit pas réalisée en autonomie, on peut observer des phénomènes de coopération entre les apprenants visant la réalisation de la tâche. Nous chercherons à déterminer des catégories d'étayage en lien avec les activités langagières définies par le Conseil de l'Europe (2001 : 18) : réception, production, interaction, médiation. Si le corpus est constitué d'interactions orales, il est possible de voir apparaître des aides spécifiques pour la réception, la production et la médiation.

Nous étudierons dans le corpus des indices d'un étayage entre apprenants en nous fondant sur l'analyse des interactions ordinaires (Traverso, 1996, 1999), l'analyse des interactions didactiques (Cicurel, 1985, 2002, 2005 ; Allwright \& Bailey, 1991 ; Bigot, 2005 ; Cambra Giné, 2003 ; Moore \& Simon, 2002) et de la communication exolingue (Bange, 1991, 1996 ; Porquier, 2004 ; Py 1993, 1996).

On peut distinguer deux types d'étayage (Bange, 1996 : 193). À un niveau global, l'étayage concerne le contexte permettant de favoriser l'autonomie des apprenants et de créer « un climat qui incite à l'initiative, à la prise de risque » (Bange, 1996 : 193). Il apparaît dans la réalisation de l'activité. C'est cependant l'étayage situé au niveau local qui fera ici l'objet de notre analyse. Il repose sur des corrections, des reformulations et des réparations (ibid.). Il correspond à «l'aide offerte par le locuteur expert lorsque l'apprenant rencontre des problèmes de compréhension ou de production dus à des lacunes dans son répertoire lexical ou éventuellement grammatical » (Mondada \& Pekarek Doehler, 2000 : 150). L'assimilation de l'étayage à ce niveau ponctuel constitue pour Mondada et Pekarek Doehler une réduction de sa portée. Cette critique ne peut toutefois pas entièrement s'appliquer à notre analyse qui portera sur les processus d'étayage entre pairs et permettra d'envisager la réciprocité de l'aide, qui n’apparaît ni comme « asymétrique », ni comme « unidirectionnel[le] » (ibid.).

\section{Manifestations de l'étayage entre pairs dans le corpus}

Trois cas de figure se présentent. Les apprenants peuvent soutenir leurs pairs dans la compréhension lexicale, jouer le rôle de médiateur auprès de l'enseignante pour assurer une intercompréhension, ou bien encore aider à la production en fournissant un mot manquant.

\subsection{Aides à la compréhension entre apprenants}

Lorsqu'un apprenant utilise un terme qui n'est pas compris par ses pairs, il peut venir en aide à ses camarades en difficulté en l'expliquant ou en le traduisant ${ }^{3}$. Ces aides à la compréhension entre étudiants témoignent d'un partage de connaissances et permettent aux apprenants de progresser grâce à leurs pairs. Afin d'assurer un accès au sens à leurs camarades, les étudiants font appel à leurs ressources plurilingues.

\subsubsection{Stratégies d'élucidation lexicale}

Les difficultés de compréhension des apprenants peuvent être gérées de différentes manières. L'enseignante n'est pas la seule détentrice du savoir: les étudiants peuvent également apporter des connaissances et transmettre un savoir culturel ou langagier. Elle est certes présente et peut fournir une aide, mais l'implication des apprenants dans la tâche permet de favoriser les élucidations lexicales entre pairs. Comme le souligne Cicurel, le professeur «lâche, par ce biais, ses prérogatives d'exper[t] pour laisser place au savoir des étudiants présents » $(2005: 188)$.

Lorsqu'une difficulté lexicale se présente, on observe l'apparition de séquences latérales explicatives :

\begin{tabular}{lll}
\multicolumn{2}{l}{ Extrait $1{\left.\text { (séquence } \mathrm{n}^{\circ} 2\right)^{4}}^{4}$} & \\
004 & Vera & euh c'est une vieille femme \\
013 & Mario & âgée femme \\
014 & $\mathrm{P}$ & une femme ÂGÉE $\uparrow$ oui \\
015 & Salma & âgée $\uparrow$
\end{tabular}




$\begin{array}{lll}016 & \text { P } & \text { oui } \\ 017 & \text { Salma } & \text { âgée } \\ 018 & \text { P } & \text { ouais } \\ 019 & \text { Salma } & \text { \#c'est quoi\# âgée } \uparrow \\ 020 & \text { P } & \text { ÂGÉE } \uparrow+\text { vieille } \\ 021 & \text { Am } & \text { XX } \\ 022 & \text { P } & \text { oui c'est ça } \\ 023 & \text { Am } & \text { vieille } \\ 024 & \text { Salma } & \text { oh } \\ 025 & \text { Cesar } & \text { c'est politesse } \\ 028 & \text { Cesar } & \text { c'est c'est poli- c'est politesse VIEUX vieux }\end{array}$

Les apprenants décrivent ici les personnages de la photographie présentée dans la séquence $\left(\mathrm{TP}^{5} 4\right)$. L'activité de description comporte nécessairement une coloration métalinguistique. Mais ici, les étudiants profitent de ce moment pour avoir recours à des essais-erreurs concernant les adjectifs. Mario reformule ainsi la proposition de Vera («c'est une vieille femme », TP 4) : «âgée femme » (TP 13). Il introduit une commutation sur l'axe paradigmatique. Salma manifeste son incompréhension en répétant plusieurs fois l'adjectif, parfois avec une intonation montante (TP 15, 17, 19). Faraco souligne le recours fréquent des apprenants de niveau débutant à la répétition du «segment non compris avec le contour intonatif du questionnement» (2002: 111). L'enseignante réagit à cette demande d'explication en établissant une relation d'équivalence entre les adjectifs «âgé » et "vieux» (TP 20). Cependant, Cesar supplée spontanément à cette élucidation lexicale lacunaire. En précisant «c'est politesse» (TP 25, 28), il ajoute une nuance pragmatique concernant la distinction entre les deux adjectifs. Dans cet extrait, la demande de Salma n'est pas adressée spécifiquement à l'enseignante ou aux apprenants.

Cependant, les étudiants peuvent s'adresser tout particulièrement à leurs pairs :

\begin{tabular}{|c|c|c|}
\hline 457 & Mario & l'abîme \\
\hline 458 & Nurhan & (petit rire) \\
\hline 459 & $\mathrm{P}$ & l'abîme $\uparrow$ \\
\hline 460 & Mario & $\underline{\text { oui }}$ \\
\hline 461 & Vera & (à Mario) qu'est-ce que c'est l'abîme \\
\hline 462 & Af & l’abîme \\
\hline 464 & Vera & $\underline{\text { hein }}$ \\
\hline 465 & As & (petit rire) \\
\hline 466 & Vera & $\underline{\text { l'abîme }}$ \\
\hline 469 & Mario & (à Vera) l'abysse l'abysse \\
\hline 470 & Vera & $\mathrm{euh}+\mathrm{AH}$ \\
\hline
\end{tabular}

Cet extrait témoigne du « support étayant entre les pairs » (Cambra Giné, 2005 : 153). Le terme « abîme » employé par Mario (TP 457) ne fait pas partie du répertoire linguistique de Vera, laquelle formule une demande de clarification linguistique à son camarade : «qu'est-ce que c'est l'abîme» (TP 461). L'apprenant s'adresse alors directement à Vera en fournissant un synonyme : «l'abysse » (TP 469). Cette élucidation lexicale semble satisfaisante pour Vera : «AH » (TP 470).

\subsubsection{Appel aux ressources plurilingues}

Pour permettre à leurs pairs d'accéder au sens, les apprenants mobilisent les langues de leur répertoire. De la même manière que les enseignants emploient comme «stratégie d'appui » (Causa, 2003: 73) «la langue que les apprenants et l'enseignant ont en commun (ou une langue véhiculaire supposée connue par le groupe) » (ibid.), les étudiants apportent leur aide à la compréhension en utilisant l'anglais :

\section{Extrait 3 (séquence ${ }^{\circ} 6$ )}

Vera (à Mario) tu peux expliquer




$\begin{array}{lll}527 & \text { Mario } & \text { quoi } \uparrow \\ 528 & \text { Vera } & \text { jure } \\ 529 & \text { Mario } & \text { jure oui *swear* } \\ 530 & \text { Nurhan } & \text { *swear* jure } \\ 531 & \text { Vera } & \text { AH:: } \\ 532 & \text { Nurhan } & \text { jure } \\ 533 & \text { Vera } & \text { ok } \\ 534 & \text { Mario } & \text { jure }\end{array}$

Cet extrait révèle les ressources des apprenants dans l'élucidation lexicale. Vera sollicite à nouveau Mario pour une clarification lexicale : «tu peux expliquer» (TP 526). Cette stratégie de demande d'aide est favorable à l'acquisition selon Cambra Giné (2003 : 138). Plutôt que d'expliquer la signification de ce verbe, l'apprenant brésilien fournit à sa camarade russe la traduction en anglais : "*swear* » (TP 529). Cette équivalence entre les deux verbes est reprise par Nurhan, une apprenante turque (TP 530). Les étudiants répètent ainsi ce nouveau verbe pour se l'approprier. De même, Mario le réemploie (TP 534), à la manière d'un professeur introduisant un nouveau terme. Tout au long de cet extrait, l'enseignante n'intervient pas : les apprenants gèrent par eux-mêmes les difficultés lexicales qui se présentent.

Comme le souligne Castellotti, en milieu homoglotte, « une autre langue partagée par l'ensemble des participants (le plus souvent l'anglais) peut jouer le rôle de langue de référence et assumer tout ou partie des fonctions généralement dévolues à cette dernière » (2001 : 58). Dans l'extrait suivant, le recours à l'anglais permet d'assurer la compréhension d'un terme français :

\begin{tabular}{|c|c|c|}
\hline \multicolumn{3}{|c|}{ Extrait 4 (séquence $\mathrm{n}^{\circ} 7$ ) } \\
\hline$\overline{119}$ & Salma & parce que c'est $\uparrow$ la femme est $\uparrow$ pri- $\uparrow$ prisonnière $\uparrow$ \\
\hline 122 & $\mathrm{P}$ & $\begin{array}{l}\text { oui (petit rire) ouais vous pensez aussi } \uparrow \text { la femme est } \\
\text { prisonnière } \uparrow\end{array}$ \\
\hline 141 & $\mathrm{P}$ & $\begin{array}{l}\text { OUI bien sûr }+ \text { Feng Yi } \uparrow \text { qu'est-ce que tu penses } \uparrow \text { tu penses } \\
\text { que la femme est prisonnière } \uparrow\end{array}$ \\
\hline 142 & Feng Yi & prisonnière $\uparrow$ \\
\hline 143 & $\mathrm{P}$ & prisonnière dans une PRISON elle est ENfermée $\uparrow$ \\
\hline 144 & Salma & euhm \\
\hline 145 & $\mathrm{P}$ & une une prison \\
\hline 149 & Feng Yi & euh \\
\hline 150 & $\mathrm{P}$ & tu comprends prison $\uparrow$ \\
\hline 152 & $\mathrm{P}$ & prison \\
\hline 153 & Cesar & *prison* \\
\hline 154 & Nurhan & *prison prisoner* \\
\hline 155 & Cesar & $*_{\text {prison }}$ break* (rires) \\
\hline 156 & Nurhan & ${ }^{* \text { prison break* }}{ }^{*}$ (rires) \\
\hline 157 & Feng Yi & AH *prison ah yes* \\
\hline 158 & As et $\mathrm{P}$ & (rires) \\
\hline 159 & $\mathrm{P}$ & OUI tu comprends (rires) \\
\hline 160 & Feng Yi & euh \\
\hline
\end{tabular}

Cependant, cet extrait diffère de celui précédemment analysé. À la suite de l'idée exprimée par Salma (TP 119), l'enseignante sollicite les apprenants pour qu'ils prennent position : «vous pensez aussi $\uparrow$ la femme est prisonnière $\uparrow »(\mathrm{TP}$ 122). Elle adresse cette question plus particulièrement à Feng Yi (TP 141). L'apprenante répète ici le mot «prisonnière $\uparrow$ » et l'emploie comme un autonyme. C'est un exemple de «stratégies de demande métalinguistique» (Cicurel, 1985: 20). L'enseignante tente d'apporter une élucidation lexicale, en mettant en évidence le mot «prison » (TP 143, 145, 150, 152), qui est susceptible de faire partie du répertoire lexical de Feng Yi. Cependant, ce terme n'est pas compris par l'apprenante. C'est alors que Cesar et Nurhan traduisent "prison » en anglais : "*prison* » (TP 153-156). Nurhan traduit également «prisonnière» (TP 154). L'énoncé de Cesar (TP 155) mérite une attention tout particulière: «*prison break*». Cette expression constitue une référence au titre d'un feuilleton 
américain. Nurhan répète cet énoncé et, tout comme Cesar, elle rit (TP 156). Il s'agit d'une stratégie culturelle d'explication qui se révèle efficace, Feng Yi comprenant finalement le sens de «prison »: «AH *prison ah yes* » (TP 157). Les stratégies déployées spontanément par les apprenants sont ici plus efficaces que celles élaborées par l'enseignante. L'exemple témoigne également de la solidarité des étudiants qui n'acceptent pas qu'un de leurs pairs ne puisse accéder au sens.

\subsection{Exercice par les apprenants du rôle de médiateur pour leurs pairs auprès de l'enseignante}

Une autre forme d'étayage entre pairs consiste à jouer le rôle le médiateur auprès de l'enseignante pour apporter une intercompréhension. Bien souvent, les apprenants comprennent leurs camarades et sont capables de transmettre le message au professeur. On se situe dans la compétence de médiation, au cours de laquelle «l'utilisateur de la langue n'a pas à exprimer sa pensée mais doit simplement jouer le rôle d'intermédiaire entre des interlocuteurs incapables de se comprendre en direct » (Conseil de l'Europe, 2001 : 71). La plupart du temps, ces locuteurs ne partagent pas la même langue. Cette compétence inclut l'interprétation, la traduction, le résumé et la reformulation dans la même langue. Porquier souligne l'importance de la «médiation informelle » (2004: 355), que l'on retrouve dans le corpus à analyser. À un niveau B2, «l'apprenant-médiateur pourra être amené à traduire, reformuler, gloser, etc. pour d'autres » (Porquier, 2004 : 356), que ce soit à partir de sa langue première ou d'une langue étrangère. Bien que les apprenants disposent seulement d'un niveau A1, ils manient déjà cette compétence avec efficacité.

Dans les deux extraits suivants, les apprenants font comprendre ce que leurs pairs veulent dire en traduisant le message en langue cible :

\begin{tabular}{|c|c|c|}
\hline \multicolumn{3}{|c|}{ Extrait 5 (séquence $\mathrm{n}^{\circ} 5$ ) } \\
\hline 019 & Salma & comment comment se dit ${ }^{*}$ sucio* \\
\hline 020 & Mario & pas propre \\
\hline 021 & $\mathrm{P}$ & pas propre $\uparrow \underline{\text { sale }} \uparrow$ \\
\hline \multicolumn{3}{|c|}{ Extrait 6 (séquence $\mathrm{n}^{\circ} 1$ ) } \\
\hline 216 & Salma & mmh: le *tren* (petit rire) \\
\hline 217 & $\mathrm{P}$ & tu dis $\uparrow$ \\
\hline 218 & Salma & le $* \operatorname{tren} *$ \\
\hline 219 & $\mathrm{P}$ & le... \\
\hline 220 & Salma & $*$ tren* \\
\hline 221 & Af & $\underline{\text { train }}$ \\
\hline 222 & $\mathrm{P}$ & $\underline{\text { le train } \uparrow}$ \\
\hline 223 & Salma & $\underline{\text { train }}$ \\
\hline
\end{tabular}

Dans l'extrait 5, Salma, une apprenante colombienne, formule une demande d'aide métalinguistique face à une lacune lexicale : "comment se dit *sucio*» (TP 19). Mario, un étudiant brésilien, traduit cet adjectif en utilisant l'autonyme précédé de la négation: «pas propre» (TP 20). Cela permet à l'enseignante de fournir l'adjectif adéquat (TP 21). Dans l'extrait 6, l'enseignante ne comprend pas le mot «*tren* » employé par Salma (TP 216-220). C'est une apprenante qui endosse alors le rôle de médiatrice, en traduisant ce terme en français (TP 221). Elle assure ainsi une intercompréhension entre Salma et l'enseignante.

Les apprenants sont également amenés à corriger les énoncés de leurs pairs afin de transmettre le message à l'enseignante :

\begin{tabular}{lll}
\multicolumn{2}{l}{ Extrait 7 (séquence $\mathrm{n}^{\circ}$ 6) } & \\
\hline 364 & Diego & mon mon rêver mon rêver rêver \\
365 & $\mathrm{P}$ & rêver $\uparrow$ \\
366 & Nurhan & mon rêve \\
367 & Vera & $\underline{\text { rêve }}$
\end{tabular}




\author{
368 Diego ah oui \\ $369 \quad \mathrm{P} \quad$ MON RÊVE OUI mon rêve $\downarrow$ euh le rêve c'est-à-dire le \\ DIVORCE $\uparrow$
}

Diego répète ici le mot "rêver » sans que l'enseignante comprenne le sens de ce terme. Nurhan et Vera déploient alors leur compétence de médiation en corrigeant la prononciation de ce terme (TP 366, 367). Cela permet à l'enseignante d'accéder au sens de l'énoncé de l'apprenant. On retrouve ce même phénomène dans l'extrait suivant, où Mario joue le rôle de médiateur en corrigeant l'énoncé de Cesar :

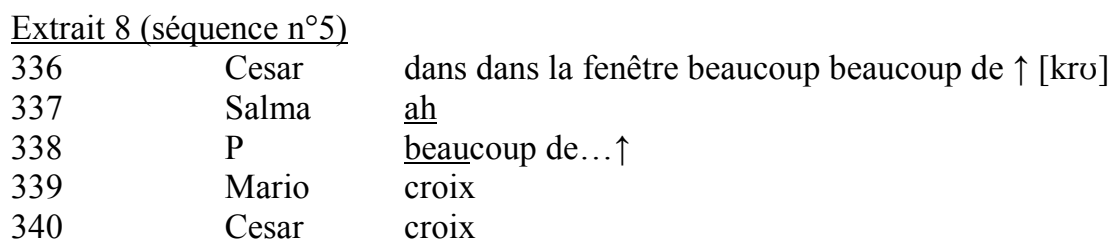

Les stratégies des apprenants mettent ainsi en évidence la «mise à contribution des savoirs et des représentations partagés » (Cambra Giné, 2003 : 138). Les obstacles à la communication sont résolus grâce à des «formes d'étayage collectif » (ibid.), ce qui crée des conditions favorables à l'acquisition.

\title{
4.3 Aide à la production
}

Si l'achèvement par les apprenants d'amorces énoncées par leurs pairs constitue une caractéristique des interactions exolingues, ce phénomène contribue également à la construction collective des interactions.

\subsubsection{La coopération lexicale}

Le dernier type d'étayage entre pairs concerne la «coopération lexicale » (Traverso, 1999: 80) : un apprenant vient en aide à un autre en apportant le mot qui lui manque, à l'occasion d'une "panne lexicale » (Traverso, 1999 : 79). Cela ne signifie pas nécessairement que ces étudiants disposent d'un niveau plus avancé que les autres. En effet, ils se situent dans un stade d'interlangue, c'est-à-dire un « ensemble plus ou moins organisé et stabilisé d[e] connaissances linguistiques » (Py, 1993: 10). Le répertoire lexical varie nettement selon les apprenants. C'est ainsi qu'ils peuvent soutenir leurs pairs pour la production orale. Les lacunes lexicales des étudiants assurent une «mise en œuvre pratique des capacités de résolution collaborative des problèmes » (Pekarek Doehler, $2000: 13$ ) et constituent à ce titre des «lieux potentiels de l'élaboration d'un répertoire variable et adapté aux besoins communicatifs pratiques » (ibid.).

Les apprenants apportent ainsi leur soutien à leurs camarades en les aidant dans l'achèvement d'un mot :

\begin{tabular}{|c|c|c|}
\hline \multicolumn{3}{|c|}{ Extrait 9 (séquence $\mathrm{n}^{\circ} 7$ ) } \\
\hline 321 & Vera & $\begin{array}{l}\text { je ne comprends pas elle euh essayer de: quoi pou- pou- } \\
\text { pousser }\end{array}$ \\
\hline 322 & Am & pousser \\
\hline 323 & Vera & pousser \\
\hline
\end{tabular}

La demande métalinguistique de Vera n'est pas explicite, mais ses pairs perçoivent ses difficultés dans la prononciation sous la forme de mots inachevés : «pou- pou- » (TP 321). On se rapproche ici de l'aide apportée par le natif pour achever l'énoncé du non-natif (Gülich, 1986 : 166). En effet, Vera complète son énoncé et ratifie par là même l'aide de ses camarades.

La coopération lexicale peut également prendre la forme de traductions en langue cible :

\begin{tabular}{lll}
\multicolumn{2}{l}{ Extrait $10\left({\text { séquence }{ }^{\circ} \text { 5) }}\right.$} & \\
\hline 045 & Cesar & il euh + euh $+*$ limpia $* \uparrow$ \\
046 & Mario & PROPRE \\
047 & Cesar & propre $\uparrow$
\end{tabular}




$\begin{array}{lll}048 & \text { Mario } & \text { propre } \\ 049 & \text { Cesar } & \text { propre les: les: les petites piscines (rires) }\end{array}$

L'énoncé de Cesar met en évidence des hésitations chez l'apprenant : « il euh + euh + *limpia* $\uparrow$ » (TP 45). L'intonation montante apparaît comme une demande linguistique. Mario apporte sa collaboration à Cesar en traduisant l'adjectif en français. Cependant, les propositions de lexique sont interprétées comme justes ou fausses, elles sont ratifiées ou non. Cesar demande une confirmation pour l'adjectif «propre » avant de l'employer (TP 47-49). Dans l'extrait suivant, Paola rencontre des lacunes au niveau lexical :

\begin{tabular}{|c|c|c|}
\hline \multicolumn{3}{|c|}{ Extrait 11 (séquence $n^{\circ} 6$ ) } \\
\hline 198 & Paola & oui oui OU euh peut-être le: la $\uparrow$ femme $\uparrow$ \\
\hline 200 & Paola & c'est:: $\uparrow$ \\
\hline 202 & Paola & c'est $\uparrow$ comment $\operatorname{dit}($ mime $«$ mort $»)$ \\
\hline 204 & Diego & mort \\
\hline 205 & Nurhan & morte \\
\hline 206 & Am & MORT \\
\hline 207 & Nurhan & $\overline{\text { elle est morte }}$ \\
\hline 208 & Diego & elle est mort \\
\hline 209 & Paola & elle elle est mort et et le homme $\uparrow$ \\
\hline 210 & $\mathrm{P}$ & oui \\
\hline 211 & Paola & est est (mime le fait d'imaginer) 1- 1- 1- \\
\hline 212 & $\mathrm{P}$ & il imagine $\uparrow$ \\
\hline 214 & Paola & non imagine la la *recall* \\
\hline 215 & Diego & rappeler \\
\hline
\end{tabular}

Sa stratégie de communication repose sur l'emploi du non-verbal: «c'est $\uparrow$ comment dit (mime «mort $) »($ TP 202). Les apprenants cherchent à lui venir en aide en fournissant le mot manquant ( «mort», TP 204-206) ou même l'énoncé complet: « elle est morte» (TP 207), « elle est mort » (TP 208). Paola ratifie l'énoncé erroné de Diego (TP 209) mais se trouve face à une nouvelle lacune lexicale pour laquelle elle a recours au mimo-gestuel (TP 211). Le verbe proposé par l'enseignante ne lui convient pas. Elle emploie alors l'anglais et Diego lui apporte le verbe approprié. Les échanges sont ici « largement orientés vers la collaboration entre pairs » (Moore \& Simon, 2002 : 124) et permettent aux apprenants de compenser leurs lacunes grâce à l'étayage de leurs camarades.

\subsubsection{La construction collective des interactions}

On peut considérer les interactions comme une «machinerie langagière » (Cicurel, 1996: 80). Klett et al. mettent en évidence le rôle de l'interaction « en tant que moteur de discours » (1994:44). Elles étudient les mécanismes qui permettent le déclenchement de la parole. L'échange est construit par l'ensemble des participants. Un premier énoncé en enclenche un autre et les apprenants alimentent les interactions. Dans les échanges à bâtons rompus ${ }^{6}$, "[1] es seules contraintes sont celles de la co-construction de l'échange verbal (pour lui-même), de la collaboration pour faciliter son développement (amplification maximale) » (Traverso, $2004: 159)$.

Lorsqu'un apprenant complète un énoncé laissé en suspens par l'un de ses pairs, on a affaire à une « interaction complémentaire» (Cicurel, 1991 : 261) avec un processus d'amorce/continuation. Cambra Giné souligne que « la rupture est signalée par des marqueurs d'incertitude surtout prosodiques (pauses, allongements, « euh:: »)»(2003 : 133). C'est le cas dans l'extrait suivant où Nurhan laisse son énoncé en suspens :

Extrait 12 (séquence $\left.{ }^{\circ} 4\right)$

$\begin{array}{lll}204 & \text { Nurhan } & \text { le mineur euh:: c'est } \uparrow \\ 205 & \text { Salma } & \text { c'est très fatigué (petit rire) } \\ 206 & \text { Nurhan } & \text { c'est très fatigué euh et très euh penseur } \uparrow\end{array}$


Le tour de parole 204 de Nurhan est marqué par des hésitations (« euh:: ») et par « une intonation montante qui indique une demande d'aide » (Cambra Giné, $2003: 147$ ) : «c'est $\uparrow$ ». Au tour de parole suivant, Salma reprend "c'est » et suggère une suite pour l'amorce de sa camarade : "c'est très fatigué " (TP 205). En répétant cet énoncé, Nurhan ratifie l'intervention de Salma qu'elle complète à son tour : " et très euh penseur $\uparrow »$ (TP 206). Cela permet ainsi à l'apprenante de développer sa propre idée. Les accords entre étudiants sont ainsi marqués par une interaction complémentaire qui indique « la continuité textuelle des échanges » (Cicurel, 1991 : 261).

Cet exemple constitue une forme de dyade : deux apprenantes s'entraident. L'échange suivant repose sur une construction plus complexe :

\begin{tabular}{|c|c|c|}
\hline \multicolumn{3}{|c|}{ Extrait 13 (séquence $n^{\circ} 6$ ) } \\
\hline 432 & Nurhan & $\underline{\mathrm{UN} \text { sur deux (petit rire) }}$ \\
\hline 433 & $P$ & un sur deux $\uparrow$ \\
\hline 434 & Nurhan & oui (rires) \\
\hline 435 & Diego & un surdeux $\uparrow$ \\
\hline 436 & Nurhan & $\underline{\mathrm{UN}}$ sur DEUX \\
\hline 437 & $\mathrm{P}$ & pourquoi un sur deux $\uparrow$ \\
\hline 438 & Nurhan & euh parce que \\
\hline 439 & Vera & $\underline{\mathbf{U N}}$ \\
\hline 440 & Nurhan & $\overline{\mathrm{UN}}$ personne mais deux euh \\
\hline 441 & Paola & personnages $\uparrow$ \\
\hline 442 & Nurhan & personnages euh \\
\hline 443 & Salma & un personne \\
\hline 444 & Nurhan & $\underline{\text { UN veut dire euh }}$ \\
\hline
\end{tabular}

On a ici affaire à un polylogue (Bouchard, 2005). L'enseignante ne constitue pas la seule locutrice ratifiée dans cet échange. Les autres apprenants témoignent verbalement de leur implication dans l'interaction. Nurhan propose d'intituler la photographie présentée «UN sur deux » (TP 432). Cet énoncé est répété en écho avec une intonation montante par l'enseignante et par Diego. L'explication de ce titre demandée par l'enseignante (TP 437) est alors construite en collaboration par les étudiants. La justification est amorcée par Nurhan : « euh parce que » (TP 438). C'est à ce moment que Vera intervient en chevauchant le tour de parole de sa camarade, qui n'a pas donné de signes indiquant qu'elle laisse la parole. L'observation de la co-construction de l'échange met ainsi en évidence des chevauchements et des interruptions, notamment avec les amorces d'énoncés complétées. Vera se contente de reprendre le premier élément du titre (TP 439). Cette intervention est validée par Nurhan qui complète son énoncé «UN personne mais deux euh » (TP 440), qu'elle laisse cependant en suspens. Paola propose un achèvement interactif avec une intonation montante : "personnages $\uparrow »(\mathrm{TP} 441)$. Nurhan accepte ce terme et le réemploie au tour de parole suivant. Une fois de plus, l'apprenante exprime son hésitation ( « euh », TP 442), ce qui permet à Salma d'intervenir : « un personne » (TP 443). Cette occurrence n'est toutefois pas ratifiée par Nurhan, dont l'intervention chevauche celle de Salma (TP 444).

Cet extrait témoigne de l'appropriation par les apprenants d'une idée émise par l'un de leurs pairs. Alors que le titre «un sur deux » a seulement été énoncé par Nurhan, l'explication de cette intitulation est construite collectivement par les étudiants. La parole circule de façon libre entre les apprenants qui complètent les énoncés de leurs pairs en maintenant une continuité thématique tout en participant à la progression de l'échange. Le Cadre Européen Commun de Référence pour les Langues précise qu'au niveau B2 avancé apparaissent des «capacités discursives [...] dans la gestion de la conversation (stratégies de coopération)» (Conseil de l'Europe, 2001 : 33). Un locuteur de niveau B2 +

\footnotetext{
est capable de donner un feed-back et une suite aux déclarations et aux déductions des autres locuteurs et, ce faisant, de faciliter l'évolution de la discussion; de mettre en relation adroitement sa propre contribution et celle des autres locuteurs (ibid.).
}

De telles compétences apparaissent ici chez des apprenants de niveau A1 ${ }^{7}$. 
Cet échange met en évidence la dynamique interactionnelle qui assure une progression. L'avancée de l'interaction nécessite « une collaboration entre les participants, elle est donc tributaire de continuels micro-accords locaux entre les participants » (Traverso, 2004 : 146). L'idée d'un apprenant en stimule ainsi un autre et les idées exprimées sont le résultat de cette co-construction. Dans le fil de la conversation, un énoncé peut, par un jeu de dominos, déclencher des résonances chez les étudiants et les encourager à procéder à des amplifications thématiques.

La coopération entre pairs peut prendre la forme d'un apprentissage entre pairs caractérisé par une entraide et un étayage entre les apprenants. Mais il importe également de souligner que les échanges directs entre les apprenants sont essentiels à la dynamique de construction collective du sens. Ils témoignent de la qualité de l'écoute, de l'attention et de la réactivité des étudiants.

\section{Conclusion}

L'analyse des interactions recueillies révèle le mode fortement coopératif sur lequel elles se déroulent. La semi-autonomie accordée aux étudiants les encourage à un apprentissage entre pairs. Nous avons relevé des phénomènes d'entraide et d'étayage visant à assurer la communication chez ces apprenants de niveau A1. La solidarité se manifeste dans les aides apportées aux étudiants en difficulté pour la compréhension ou la production. Les apprenants s'exercent ce faisant au rôle de médiateur et éprouvent le même besoin de transmettre les idées de leurs camarades que s'il s'agissait des leurs. Le savoir est circulant, ce qui permet aux étudiants de participer à l'élaboration des connaissances et aux processus d'apprentissage. Les apprenants interagissent ainsi comme s'ils travaillaient en groupes autonomes.

Ne disposant pas de données permettant de procéder à une analyse acquisitionnelle à travers une étude longitudinale, nous ne pouvons pas mesurer l'acquisition effective des apprenants. Cependant, dans une approche socioconstructiviste, nous pouvons considérer que les interactions dans l'activité réunissent des conditions propices à l'appropriation langagière.

Le phénomène d'étayage entre pairs, orienté vers une autonomisation des étudiants, semble favorisé par l'attitude de l'enseignante qui intervient peu. Il peut également être attribué aux « enjeux communicatifs » (Moore \& Simon, 2002) suscités par la tâche de commentaire de photographies (Muller, 2012a). Les images, en interpelant les apprenants, créent des besoins de dire et incitent ainsi les locuteurs à des stratégies de prise de risque nécessaires à la progression (Muller, 2012b).

Nous avons ici analysé des extraits dans lesquels l'étayage entre les apprenants réussit. Il serait intéressant de chercher dans les interactions transcrites des échecs ou des malentendus en la matière. De même, il conviendrait de s'interroger sur les aspects pragmatiques de tels échanges. Certes, un climat de solidarité peut régner entre les étudiants, mais des enjeux de positionnement discursif ou de figuration sont également susceptibles d'apparaître. Dans une étude ultérieure, l'étayage entre pairs pourrait être considéré sous l'angle de la pragmatique des interactions. Celui qui offre son aide à l'autre en lui permettant d'accéder au sens ou en produisant un énoncé à sa place n'usurpe-t-il pas la place de l'autre ? Il se situe en tout cas dans une place haute d'enseignant et renvoie son interlocuteur à une position basse d'apprenant. Aider son camarade en difficulté permet-il à celui-ci de ne pas perdre la face ou, au contraire, aider un pair - et témoigner ainsi de sa supériorité - ne contribue-t-il pas à menacer la face de son interlocuteur, en mettant en évidence ses lacunes? Une analyse minutieuse des enjeux de contenu permettrait de répondre à ces interrogations.

\section{Références bibliographiques}

Allwright, D. \& Bailey, K. M. (1991). Focus on the Language Classroom. An introduction to classroom research for language teachers. Cambridge : Cambridge University Press.

Arborio, A.-M. \& Fournier, P. ([1999] 2010). L'enquête et ses méthodes, l'observation directe. Paris : Armand Colin.

Bange, P. (1991). Séquences acquisitionnelles en communication exolingue. In Russier, C., Stoffel, H. \& Véronique, D. (éd.) Interactions en langue étrangère. Aix-en-Provence : Publications de l'université de Provence, 61-66. 
Bange, P. (1992). À propos de la communication et de l'apprentissage de L2 (notamment dans ses formes institutionnelles). Acquisition et Interaction en Langue Étrangère, $n^{\circ} 1,53-85$.

Bange, P. (1996). Considérations sur le rôle de l'interaction dans l'acquisition d'une langue étrangère. Les Carnets du CEDISCOR, $n^{\circ} 4,189-202$.

Beacco, J.-C. (2008). Chapitre 2: Spécifications générales du Niveau A2 pour le français : de la compétence de communication au répertoire de genres discursifs de l'utilisateur/apprenant. In Beacco, J.-C, Lepage, S., Porquier, R. \& Riba, P. (éd.) Niveau A2 pour le français. Un référentiel. Paris : Didier, 23-48.

Bigot, V. (2005). Quelques questions de méthodes pour une recherche sur la construction de la relation interpersonnelle en classe de langue : primauté des données et construction de savoirs. Le Français dans le monde. Recherches et applications, $n^{\circ}$ spécial, juillet 2005, 42-53.

Blanchet, P. (2000). La linguistique de terrain. Méthode et théorie. Une approche ethno-sociolinguistique. Rennes : Presses Universitaires de Rennes.

Blanchet, P. (2011). L'observation participante. In Blanchet, P. \& Chardenet, P. (éd.) Guide pour la recherche en didactique des langues et des cultures. Approches contextualisées. Paris : Éditions des archives contemporaines \& Montréal : Agence Universitaire de la Francophonie, 73-74.

Bouchard, R. (2005). Les interactions pédagogiques comme polylogues, Lidil $n^{\circ} 32,139-156$

Bruner, J. S. (1983). Le développement de l'enfant : savoir faire, savoir dire. Paris : Presses Universitaires de France.

Cambra Giné, M. (2003). Une approche ethnographique de la classe de langue. Paris : Didier.

Cambra Giné, M. (2005). L'(auto-)observation et l'analyse de l'interaction en sous-groupes par de futurs enseignants de FLE : l'évolution des représentations. Le Français dans le monde, Recherches et applications, $n^{\circ}$ spécial, juillet 2005, 148-159.

Castellotti, V. (2001). La langue maternelle en classe de langue étrangère. Paris : Clé international.

Causa, M. (2003). Les langues maternelles comme support à la transmission de savoirs et de savoir-faire en langue étrangère. Triangle, $n^{\circ} 19,67-82$.

Cicurel, F. (1985). Parole sur parole ou le métalangage dans la classe de langue. Paris : Clé international.

Cicurel, F. (1991). L'identité discursive d'un apprenant en langue. In Russier, C., Stoffel, H. \& Véronique, D. (éd.) Interactions en langue étrangère. Aix-en-Provence : Publications de l'Université de Provence, 259-269.

Cicurel, F. (1996). L'instabilité énonciative en classe de langue : du statut didactique au statut fictionnel du discours. Les Carnets du CEDISCOR, $n^{\circ} 4,77-92$.

Cicurel, F. (2002). La classe de langue un lieu ordinaire, une interaction complexe. Acquisition et Interaction en Langue Étrangère, $n^{\circ} 16,145-163$.

Cicurel, F. (2005). La flexibilité communicative. Un atout pour la construction de l'agir enseignant. Le Français dans le monde, Recherches et applications, $n^{\circ}$ spécial, juillet 2005, 180-191.

Cicurel, F. (2011). " Les interactions en situation d'enseignement-apprentissage : observer, transcrire, analyser ». In Blanchet, P. \& Chardenet, P. (éd.) Guide pour la recherche en didactique des langues et des cultures. Approches contextualisées. Paris: Éditions des archives contemporaines \& Montréal: Agence Universitaire de la Francophonie, 323-335.

Conseil de l'Europe (2001). Un cadre européen commun de référence pour les langues: apprendre, enseigner, évaluer. Paris : Didier.

Cots, J. M., Baiget, E., Irun, M., Llurda, E. \& Arno, E. (1997). Modes de résolution de tâches métalinguistiques en travail de groupe. Acquisition et Interaction en Langue Étrangère, $n^{\circ} 10,75-106$

Donato, R. (1994). Collective scaffolding in second language learning. In Lantolf, J. P., Appel, G. (éd.) Vygotskian Approaches to Second Language Research, New Jersey : Ablex, 33-56.

Ellis, R. ([1994] 2008). The Study of Second Language Acquisition. Oxford : Oxford University Press.

Faraco, M. (2002). Répétition, acquisition et gestion de l'interaction sociale en classe de L2. Acquisition et Interaction en Langue Étrangère, $n^{\circ} 16,97-120$. 
Gülich, E. (1986). L'organisation conversationnelle des énoncés inachevés et de leur achèvement interactif en situation de contact. DRLAV, $n^{\circ} 34-35,161-182$

Hugon, M.-A. \& Le Cnuff, C. (éd.) (2011). Interactions dans le groupe et apprentissages. Nanterre: Presses Universitaires de Paris Ouest.

Klett, E., Lucas, M., Scipioni, L. \& Vidal, M. (1994). Pour une recherche des positions discursives : l'enseignant et l'apprenant en situation d'interview. Les Carnets du CEDISCOR, $n^{\circ} 2,35-48$.

Lantolf, J. P. \& Genung, P. B. (2000). L'acquisition scolaire d'une langue étrangère vue dans la perspective de la théorie de l'activité : une étude de cas. Acquisition et Interaction en Langue Étrangère, $n^{\circ} 12$, 99-122.

Mondada, L. \& Pekarek Doehler, S. (2000). Interaction sociale et cognition située : quels modèles pour la recherche sur l'acquisition des langues? Acquisition et Interaction en Langue Étrangère, $n^{\circ} 12,147-174$.

Moore, D. \& Simon, D. L. (2002). Déritualisation et identité d'apprenants. Acquisition et Interaction en Langue Étrangère, Vol. 16, 121-143.

Muller, C. (2011a). Paroles sur images: Les interactions orales déclenchées par des photographies d'auteur en classe de français, langue étrangère. Thèse de doctorat, Paris : Université Sorbonne Nouvelle - Paris 3.

Muller, C. (2011b). Effet d'une activité inhabituelle sur le positionnement de l'enseignant et des apprenants : vers une inversion des rôles? Colloque international Spécificités et diversité des interactions didactiques : disciplines, finalités, contextes, Publication sur le site HAL SHS : http://hal.archivesouvertes.fr/docs/00/53/37/86/PDF/MULLER.pdf.

Muller, C. (2012a). La photographie, un outil pour communiquer en classe de langue. Recherche et pratiques pédagogiques en langues de spécialité, Cahiers de l'APLIUT Volume XXXI n¹. 10-27.

Muller, C. (2012b). Compétence d'interaction orale et stratégies des apprenants : comment favoriser la progression à travers une tâche communicative. In Valetopoulos, F. \& Zajak, J. (éd.) Les compétences en progression. Varsovie, Presses de l’Université de Varsovie. À paraître.

Muller, C. (2012c). Genres interactifs oraux et niveaux du Cadre. Pour des profils différenciés de compétences. In Stratilaki, S. \& Fouillet, R. (éd.) Hommages à Jean-Claude Beacco. Paris : Riveneuve Éditions. À paraître.

Nussbaum, L. (1999). Émergence de la conscience linguistique en travail de groupe entre apprenants de langue étrangère. Langages, $n^{\circ} 134,35-50$.

Pekarek Doehler, S. (2000). Approches interactionnistes de l'acquisition des langues étrangères: concepts, recherches, perspectives. Acquisition et Interaction en Langue Étrangère, $n^{\circ}$ 12, 3-26.

Porquier, R. (2004). Stratégies d'apprentissage. In Beacco, J.-C., Bouquet, S. \& Porquier, R. (éd.), Niveau B2 pour le français. Un référentiel. Paris : Didier, 353-360.

Py, B. (1993). L'apprenant et son territoire : système, norme et tâche. Acquisition et Interaction en Langue Étrangère, $n^{\circ} 2,9-24$.

Py, B. (1996). Les données et leur rôle dans l'acquisition d'une langue non maternelle. Les Carnets du CEDISCOR, $n^{\circ} 4,95-110$.

Soulé, B. (2007). Observation participante ou participation observante ? Usages et justifications de la notion de participation observante en sciences sociales. Recherches qualitatives, $n^{\circ} 27$ (1), 127-140.

Springer, C. (2002). Préface. In Helmling, B. (éd.) L'apprentissage autonome des langues en tandem. Paris : Didier, $7-9$.

Springer, C. (2009). La dimension sociale dans le CECR : pistes pour scénariser, évaluer et valoriser l'apprentissage collaboratif. Le Français dans le Monde Recherches et applications, $n^{\circ}$ 45, 25-34.

Traverso, V. (1996). La conversation familière. Analyse pragmatique des interactions. Lyon : Presses Universitaires de Lyon.

Traverso, V. (1999). L'analyse des conversations. Paris : Nathan.

Traverso, V. (2004). Grille d'analyse des discours interactifs oraux. In Beacco, J.-C., Bouquet, S. \& Porquier, R. (éd.) Niveau B2 pour le français (utilisateur / apprenant indépendant). Textes et références. Strasbourg : Division des Politiques linguistiques et Paris : Didier, 119-148. 
van Lier, L. ([1988] 1990). The Classroom and the Language Learner. Ethnography and Second-Language Classroom Research. Londres : Longman.

van Lier, L. (1996). Interaction in the Language Curriculum. Awareness, Autonomy and Authenticity. Londres: Longman.

Vygotski, L. ([1933] 1997). Pensée et langage. Paris : La Dispute.

\footnotetext{
${ }^{1}$ Cette expression se trouve dans la traduction de F. Sève (1997). Ce concept est également désigné par «zone proximale de développement » ou « zone de développement proximal ».

${ }^{2}$ Afin de distinguer nos rôles d'enseignante et de chercheure, nous emploierons la troisième personne lorsque nous nous réfèrerons à nous-même en tant qu'enseignante.

${ }^{3}$ Ce phénomène est susceptible d'apparaître dans les interactions enregistrées. Il ne s'agit cependant pas d'une règle communicative observée dans l'ensemble des interactions didactiques.
}

${ }^{4}$ Conventions de transcription :

$\begin{array}{ll}\text { AH } & \text { emphase intonative } \\ \text { pol- } & \text { mot tronqué } \\ \text { femme } & \text { chevauchement } \\ \text { *yes* } & \text { segment dans une langue autre que le français } \\ {[\mathrm{krv}]} & \text { transcription en alphabet phonétique international } \\ \text { \#quoi\# } & \text { segment incertain } \\ \text { XXX } & \text { segment incompréhensible (le nombre de X correspond au nombre de syllabes) } \\ \text { (rires) } & \text { commentaire sur le non-verbal ou le para-verbal } \\ : & \text { allongement de la syllabe } \\ :: & \text { allongement plus long de la syllabe } \\ + & \text { pause (1 à } 2 \text { secondes) } \\ \uparrow & \text { intonation montante } \\ \downarrow & \text { intonation descendante } \\ \ldots \uparrow & \text { demande d'achèvement interactif } \\ \text { P } & \text { professeur } \\ \text { Vera } & \text { prénom de l'apprenant quand identifié } \\ \text { Af } & \text { apprenante non identifiée } \\ \text { Am } & \text { apprenant (homme) non identifié } \\ \text { As } & \text { plusieurs apprenants }\end{array}$

Les prénoms des apprenants ont été modifiés en tenant compte de leur sexe et de leur nationalité.

L'utilisation de caractères gras vise à mettre en évidence le phénomène observé. Les segments en gras sont ceux qui font plus particulièrement l'objet de notre attention dans l'analyse.

${ }^{5} \mathrm{TP}=$ tour de parole

${ }^{6}$ Certains extraits du corpus se rapprochent de la conversation à bâtons rompus : l'interaction peut être ponctuellement autogérée par les apprenants. Ceux-ci échangent sur un ton léger et ludique, en ayant recours à des plaisanteries et à des glissements thématiques. 
${ }^{7}$ Ce phénomène témoigne de l'écart entre les compétences attendues pour un niveau de compétences et le niveau de performance effectif des locuteurs. Ce «paradoxe didactique» (Muller, 2012c) plaide en faveur de "profils différenciés de compétences » (Beacco, 2008) 